\title{
Measurement Accuracy Enhancement via Radio Frequency Filtering in Distributed Brillouin Sensing
}

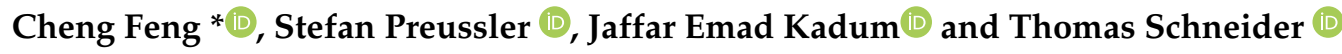 \\ THz Photonics Group, Institut für Hochfrequenztechnik, Technische Universität Braunschweig \\ Schleinitzstr. 22, 38106 Braunschweig, Germany \\ * Correspondence: cheng.feng@ihf.tu-bs.de; Tel.: +49-531-391-2011
}

Received: 12 June 2019; Accepted: 26 June 2019; Published: 28 June 2019

\begin{abstract}
In this article, we demonstrate the noise reduction and signal to noise ratio (SNR) enhancement in Brillouin optical time-domain analyzers (BOTDA). The results show that, although the main noise contribution comes from the Brillouin interaction itself, a simple low pass filtering on the detected radio frequency (RF) signal reduces remarkably the noise level of the BOTDA traces. The corresponding SNR enhancement depends on the employed cut-off frequency of the low pass filter. Due to the enhancement of the SNR, a mitigation of the standard deviation error of the Brillouin frequency shift (BFS) has been demonstrated. However, RF filters with low cut-off frequency could lead to distortions on the trace signals and therefore detection errors on a non-uniform BFS. The trade-off between the noise reduction and the signal distortion as well as an optimal cut-off frequency are discussed in detail.
\end{abstract}

Keywords: stimulated brillouin scattering; Brillouin optical time-domain analyzers; fiber optics sensors; distributed optical sensing; radio frequency filtering

\section{Introduction}

Distributed sensing applications such as the Brillouin optical time-domain analysis (BOTDA) have attracted immense interest in recent years in fields like health monitoring of large structures in oil and gas pipelines, railways and high voltage transmission lines [1,2], high temperature distributed measurement in industrial applications [3], distributed strain measurement for cracks detection and monitoring [4] and security intrusion monitoring over long fences [5]. BOTDA sensors exploit the stimulated Brillouin scattering (SBS) in optical fibers, that initiates after a pulsed pump wave counter-propagates with a probe continuous wave (CW) which leads to an excitation of an acoustic wave. The SBS interaction leads to a frequency down-shifted gain region where a counter propagating wave is amplified and a frequency up-shifted loss region where the counter propagating wave is attenuated. Based on this interaction, the energy is transferred from the higher frequency pump wave to the probe wave via the acoustic wave, leading to a depletion of the pump signal and a Brillouin gain for the probe signal. The efficiency of this energy transfer depends on the frequency offset between the pump and probe wave, which is called Brillouin frequency shift (BFS) and around $11 \mathrm{GHz}$ in standard single mode fibers (SSMF) [6]. This BFS is sensitive to both temperature and strain in the optical fiber, which makes the basic principle of the BOTDA [7]. In order to accurately estimate the BFS, the Brillouin gain spectrum (BGS) is measured at each fiber location by sweeping the pump or probe frequency offset [7] and reconstructed by fitting with the theoretical profile $[8,9]$. However, the gain in a pulse-CW interaction is limited due to the short interaction length, which is determined by the spatial resolution requirement and reduces the signal to noise ratio (SNR) of the detected probe signal drastically. Since for a conventional BOTDA system, i.e., single pulse pump wave, single sideband probe $\mathrm{CW}$ without fiber loop configuration as in the first experimental demonstration [10] and defined 
as the standard scheme in [11], the pump wave is constrained by modulation instability (MI) [12] and the probe power is limited by nonlocal effects [13], the power of the pump and probe wave cannot be increased to enhance the SNR.

The accuracy of the BFS estimation is the key performance of the BOTDA sensing. The estimation error in the experiment can be statistically determined by the standard deviation of the measured BFS after a large number of consecutive measurements. It depends on the full-width at half maximum (FWHM) of the BGS $\Delta v_{B}$, the frequency scanning step $\delta$ and the system noise $\sigma$ at the location of the fiber section, which is the inverse of the system SNR [11]. Therefore, noise reduction has a direct impact on the enhancement of the accuracy of the BFS estimation.

Many methods have been proposed to increase the SNR in BOTDA sensors. The SNR can be enhanced by increasing the number of averages of the data traces, however, this comes at the cost of an increased measurement time. Other methods like differential $\pi$-phase-shifted pulse pair [14], Raman amplification [15], self-heterodyne detection [16], optical pulse coding [17], wavelet de-noising techniques [18] and 2D and 3D image restoration [19] have been proposed as well. However, these methods complicate either the experimental setup or the post-processing algorithm. Here, a very simple and direct method for the noise reduction in the detected probe signal, based on radio frequency (RF) low pass filtering, is presented. Since the thermal, shot and dark noise from a detector, and the relative intensity noise (RIN) from the laser source $[16,20]$, are proportional to the square root of the system bandwidth $B_{s y s}$ [21], RF low pass filtering could theoretically suppress these noise contributions in a BOTDA system by a factor of $\sqrt{B_{s y s} / B_{f}}$, where $B_{f}$ is the filter bandwidth. However, the main noise contribution in a Brillouin amplifier comes from the Brillouin interaction itself [22,23]. This contribution cannot simply be filtered out. However, as we will show in the following, a simple filter bandwidth reduction has a significant effect on the BOTDA sensor performance.

\section{Simulation}

\subsection{Theory and Modeling}

For a CW-SBS interaction, the complex BGS profile is given by [24]

$$
G_{B}(\omega)=\frac{\frac{1}{2} g_{0} P_{p}}{1-2 j\left(\omega-\omega_{B}\right) / \Gamma_{B}}
$$

where $g_{0}$ is the SBS gain coefficient, $P_{p}$ is the pump power launched into the fiber, $\omega_{B} / 2 \pi$ is the BFS and $\Delta v_{B}=\Gamma_{B} / 2 \pi$ is the SBS gain bandwidth, which is usually in the range of 20 to $30 \mathrm{MHz}$ in SSMF at $1550 \mathrm{~nm}$ pump wavelength and can be reduced by one order of magnitude with several methods for CW-SBS interactions [25-28]. The imaginary part of Equation (1) is the Brillouin phase spectrum and the real part represents the BGS which is approximated by a Lorentzian shape [24]. Provided that the pump depletion is negligible and the extinction ratio (ER) of the pump pulse is infinitely high so that no pump leakage-probe interaction is considered [29-31], the local Brillouin gain $g_{B}$ that will be experienced by the probe wave at position $z$ is given by

$$
g_{B}(\omega, z)=\exp \left[G_{B}(\omega) L_{e f f}\right] \cdot \exp [-2 \alpha z]
$$

where $\alpha$ is the linear loss coefficient of the fiber, $L_{e f f}=[1-\exp (-\alpha L)] / \alpha$ represents the effective length, $L=v_{\mathcal{C}} T / 2$ has the same expression as the spatial resolution, where $v_{\mathcal{C}}$ is the speed of light in the fiber and $T$ is the pulse width.

In BOTDA sensors, a pulse train is launched into the fiber as the pump signal with a pulse width $T$ and peak pulse power $P_{p}$. Thus, each pump pulse interrogates the fiber sections sequentially and consequently, the CW probe wave at different fiber sections will experience different Brillouin gain, generating time resolved traces, according to the pulse train repetition rate. Then the frequency domain representation of the detected BOTDA signal is obtained by applying a Fourier transformation (FT) 
to the detected BOTDA traces. Please note that, the FT of the BOTDA probe signal in the frequency domain determines the required bandwidth of the photodiode (PD) while the BGS is scanned by the pump-probe frequency difference.

In an additive white Gaussian noise channel (AWGN), the noise power is directly proportional to the bandwidth. Thus, noise reduction and SNR enhancement can be performed by removing (filtering) noisy spectral components out of the detected RF spectrum of the probe signal. Let $H(\omega)$ be the filter transmission response, $P_{S}(\omega)$ the FT of the time-varying probe traces and $P_{\text {out }}(\omega)$ the RF filtered output spectrum, then

$$
P_{\text {out }}(\omega)=P_{S}(\omega) H(\omega)
$$

The filtered time domain probe traces can be obtained by applying the inverse FT to $P_{\text {out }}(\omega)$

$$
P_{\text {out }}(t)=\mathcal{F}^{-1}\left[P_{\text {out }}(\omega)\right]
$$

where $\mathcal{F}^{-1}$ denotes the inverse FT.

\subsection{Frequency and Time Domain Simulation}

The simulation is carried out based on the simple model presented in Section 2.1. In BOTDA, the Brillouin gain experienced by the probe signal is calculated according to Equations (1) and (2) at each fiber section. The BGS is calculated with $200 \mathrm{MHz}$ frequency span and $1 \mathrm{MHz}$ step. The repetition rate and pulse width of the pump pulse in the simulation are $8.3 \mathrm{kHz}$ and $100 \mathrm{~ns}$, respectively (same values as for the experiment). The spatial resolution $\Delta z$ is $10 \mathrm{~m}$ which is determined by the pump pulse width $(100 \mathrm{~ns})$ and is given by $v_{c} T / 2$, where $v_{c}$ is the speed of light in the fiber and $T$ is the pulse width. The probe and pump power are set to $-14 \mathrm{dBm}$ and $18 \mathrm{dBm}$, respectively. In order to study the effect of the RF filtering, random noise is added to the traces. For a fair comparison, the noise level on all the traces in the simulation are the same. The spectra of the traces are obtained by applying a fast FT to the time domain traces. The filtering effect is simulated by multiplying the spectra with the transfer function of a RF low pass filter. The filtered time domain traces are retrieved by applying inverse fast FT to the filtered spectrum. The SBS linewidth is $50 \mathrm{MHz}$, while the assumed BFS is $10.615 \mathrm{GHz}$ and the total fiber length is $10 \mathrm{~km}$. Figure 1a shows the time domain traces of the probe wave for the ideal case, i.e., without any noise, for the conventional case, i.e., with noise (it refers to a direct detection at the full detector bandwidth without employing any additional filters in the experiment), and for low pass filtered cases with cut-off frequencies of 2, 10 and $25 \mathrm{MHz}$. As it is clearly illustrated, the low pass filters reduce the noise, i.e., the lower the filter bandwidth is, the lower the noise will be.

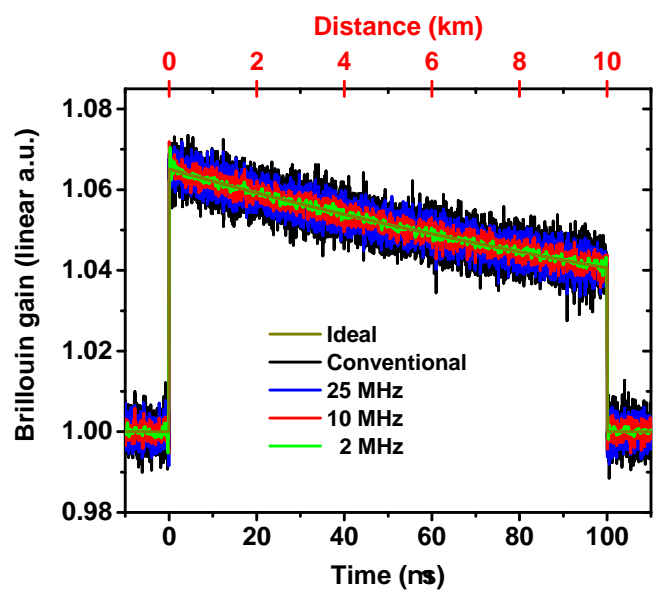

(a)

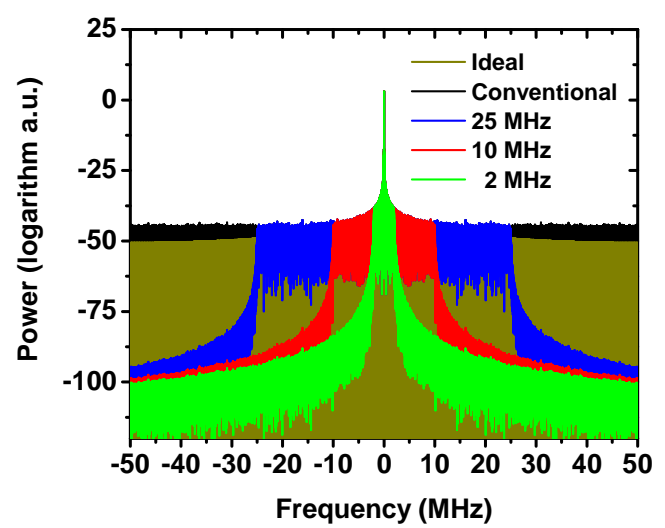

(b)

Figure 1. (a) Simulated time domain traces of the ideal, conventional and filtered probe signal; (b) simulated spectrum of the detected radio frequency (RF) ideal, conventional and filtered probe signal with different filter bandwidths. 
The ideal spectrum in Figure $1 \mathrm{~b}$ represents the frequency spectrum of the detected RF trace signal without any noise (dark yellow curves in both Figure 1a,b) while the black one represents the spectrum of a conventional BOTDA signal after applying noise but without employing any filtering. As expected, using low pass filtering rejects the out-of-band noise components and therefore reduces the noise of the time domain traces.

\subsection{Measurement Accuracy}

The measurement accuracy, which plays a vital role in the evaluation of sensor performance, symbolizes the uncertainty of the measurand (temperature or strain). It is characterized by the BFS estimation error in a BOTDA system and highly dependent on the probe SNR. As the reconstructed BGSs in Figure 2a depicts, the BGS with a lower bandwidth filter is clearer and makes the BFS estimation easier, which shows coherence to the time domain trace results in Figure 1a. If the noise is defined as the the root mean square (RMS) amplitude of the BGS in the frequency domain [11], the noise level for the $2 \mathrm{MHz}, 10 \mathrm{MHz}$ and $25 \mathrm{MHz}$ filter are $29.8 \%, 64.1 \%$ and $99.8 \%$ compared to the conventional case. Since the pulse width in the simulation is $100 \mathrm{~ns}$ and hence its BGS is more Lorentzian-like [32], the BFS along the fiber is reconstructed by a Lorentzian fitting of the measured BGS. In the simulation, the standard deviation is calculated from a set of 200 BFS measurements at each fiber segment. Figure $2 b$ illustrates the standard deviation error. The ideal trace, which is considered without any noise, has a zero standard deviation error, while the curve with too large bandwidth (with noise but without applying proper low pass filtering) has the highest standard deviation error. Note that the conventional error curve and the curve after applying the $25 \mathrm{MHz}$ filter are well overlapped, since the $25 \mathrm{MHz}$ filter allows still higher noise frequency components and thus lower SNR. The standard deviation error is decreased when the cut-off frequency of the filter is reduced. The standard deviation error at the end of the fiber with $2 \mathrm{MHz}$ filtering is about only $50 \mathrm{kHz}$ compared to $210 \mathrm{kHz}$ when a $25 \mathrm{MHz}$ cut-off frequency is used. Since the pump power is reduced exponentially along the fiber due to the fiber losses, the standard deviation error is increased exponentially.

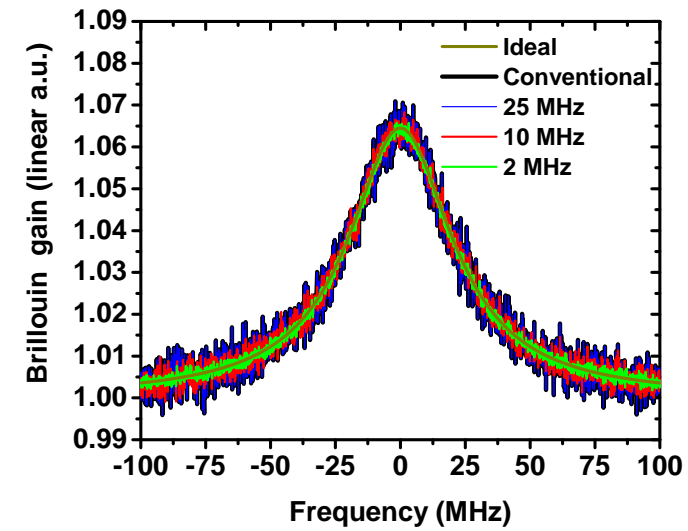

(a)

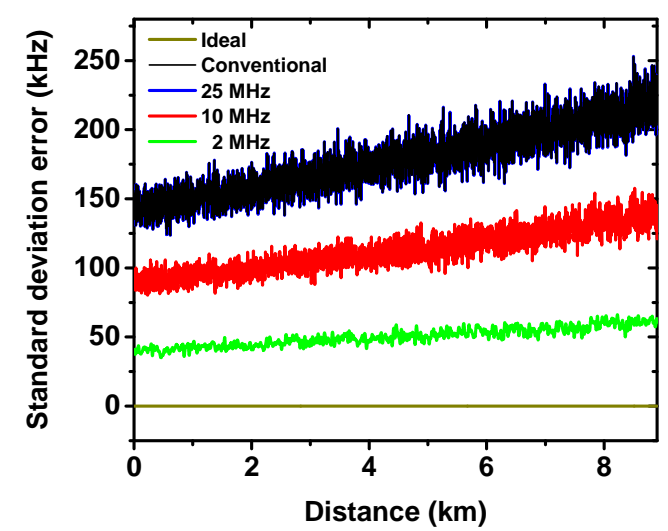

(b)

Figure 2. (a) Reconstructed Brillouin gain spectrum (BGS) (resolution $0.1 \mathrm{MHz}$ ) at the near end of the fiber and (b) standard deviation error after applying RF filtering with different bandwidths in the simulation.

\section{Experiment and Results}

\subsection{Experimental Setup}

Figure 3 shows the experimental setup, the output from the DFB-laser at $1550 \mathrm{~nm}$ is split into two branches via a 90/10 optical coupler. In the lower branch, the optical pulses are generated using a switching type semiconductor optical amplifier (SOA) (Thorlab SOA1013SXS switched by a Highland 
Technology T160-9 driving board) driven by an electrical pulse sequence with $8.33 \mathrm{kHz}$ repetition rate to get high ER (32 dB) optical pulses. The optical pulses are amplified by an erbium-doped fiber amplifier (EDFA) (Photop Koncent, Inc. PTEDFA-PA-C-SCH-15-FC/APC with constant current mode) to $14 \mathrm{dBm}$ to avoid MI [12]. Though the pump power is not optimized to the best performance, a better frequency error improvement could be demonstrated with this power level. To compensate the polarization fading, a polarization scrambler (General Photonics PSY-101) was used before directing the pump pulses into the fiber via an optical circulator. In the upper branch, the double-side band probe wave was formed by modulating the laser via a Mach Zehnder modulator (MZM) driven by an RF generator (Anritsu MG3692C), which scans the RF frequency with a span of $150 \mathrm{MHz}$ and $1 \mathrm{MHz}$ step to reconstruct the BGS. The bias point of the MZM was set to get a probe signal with double sideband and suppressed-carrier to generate dual (gain and loss) Brillouin interaction that compensates each other to reduce pump depletion [33]. The power of the probe wave was set to $-14 \mathrm{dBm}$ to avoid nonlocal effects [13]. After SBS interaction between pump and probe waves, the BOTDA signal was filtered by a narrowband Fiber Bragg Grating (FBG, AOS T-FBG) to remove the upper sideband of the probe wave. The RF probe signal was detected by a PD (Optilab PD-20)—with an electrical bandwidth of $20 \mathrm{GHz}$ and a responsitivity of $0.85 \mathrm{~A} / \mathrm{W}$ - and passed through a commercial low pass filter. After that, the probe signal was received by a digitizer (Acqiris U5309A, $300 \mathrm{MHz}$ bandwidth) with a sample rate of $1 \mathrm{GSa} / \mathrm{s}$ and 8 bits resolution and computer for averaging and further analysis.

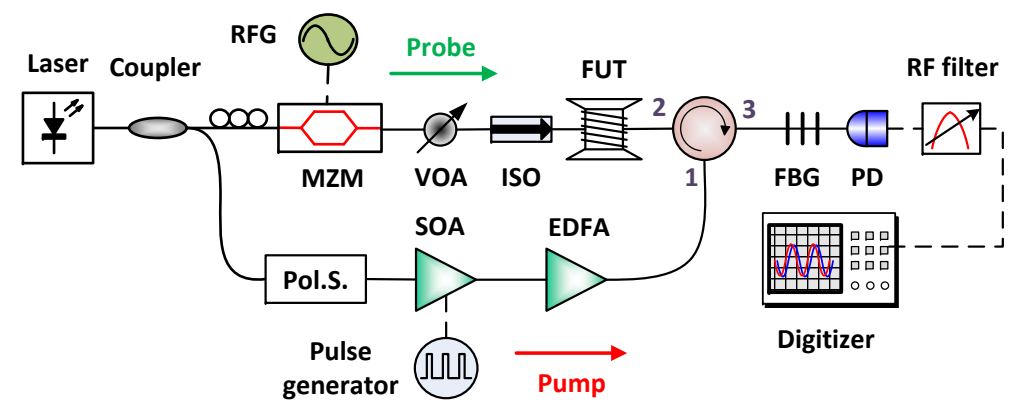

Figure 3. Experimental setup: RFG: radio frequency generator, MZM: Mach Zehnder modulator, VOA: variable optical attenuator, ISO: isolator, FUT: fiber under test, CIR: circulator, FBG: fiber Bragg grating, Pol.S: polarization scrambler, SOA: semiconductor optical amplifier, EDFA: Erbium-doped fiber amplifier, PD: photodiode.

\subsection{Results}

First, we study the effect of the RF filtering on the time domain traces in regard to noise reduction, Figure 4a illustrates the RF spectra measured by an electrical spectrum analyzer (ESA). As it can be seen, the spectrum is reduced depending on the cut-off frequency of the used low pass filter. Figure $4 \mathrm{~b}$ shows the corresponding time domain traces measured by a digitizer, the effect of RF filtering can be seen from the noise level mitigation on these traces. According to the conventional definition of RMS noise in the frequency domain [11], the noise level for $2 \mathrm{MHz}, 10 \mathrm{MHz}$ and $25 \mathrm{MHz}$ are $65.7 \%, 78.9 \%$ and $94.7 \%$ compared to the conventional case, respectively. As clearly demonstrated, the noise suppression via low pass filtering in the experiment is, though remarkable, lower than in the simulation, especially for low filter bandwidths ( $2 \mathrm{MHz})$. This indicates that, the noise in the BOTDA system is not a pure AWGN channel. With a simple filter, the bandwidth dependent AWGN can be efficiently suppressed but the gain dependent SBS noise cannot. 


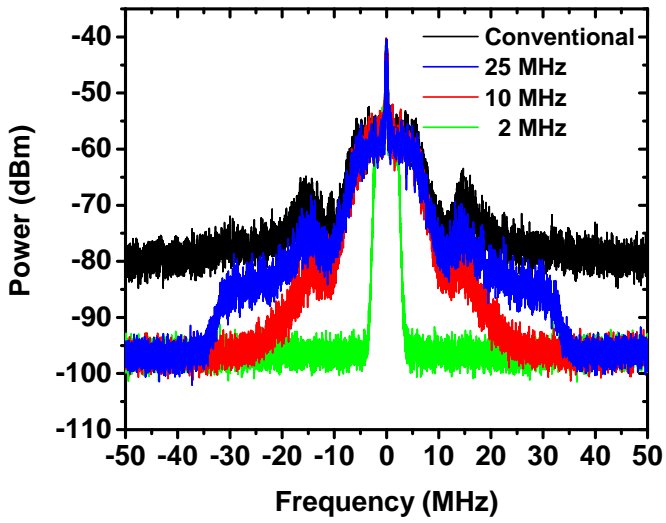

(a)

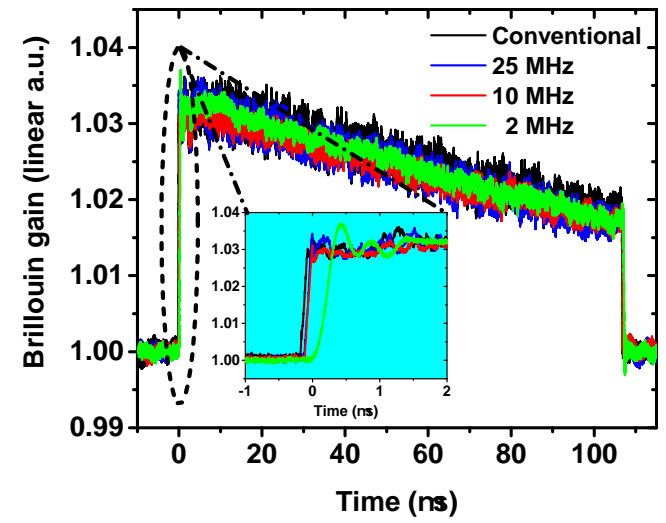

(b)

Figure 4. Experimental measurement results using RF low pass filtering: (a) RF spectrum measured by ESA; (b) time domain traces measured by a digitizer. The inset is the zoom in of the trace at the near end of the fiber.

Next, the effect of the RF filtering on the BOTDA accuracy was evaluated. Figure 5a shows the evolution of the standard deviation error along the fiber. Due to the noise reduction and SNR enhancement, the impact of the RF filter can be clearly observed. The error is $208 \mathrm{kHz}$ at $10 \mathrm{~km}$ distance with a $25 \mathrm{MHz}$ filter, which corresponds to a measurand (temperature) resolution of $0.2{ }^{\circ} \mathrm{C}$, while it is reduced to $147 \mathrm{kHz}$ (measurand resolution $0.15^{\circ} \mathrm{C}$ ) with a $2 \mathrm{MHz}$ filter. Meanwhile, applying $2 \mathrm{MHz}$ filtering reduces the standard deviation error by $32 \%$ compared to the conventional case.

At last, the figure of merit (FoM) [11] as a criteria of the sensing performance is calculated to be $0.0277,0.0316,0.0348$ and 0.0387 for the conventional case and for the applied filter bandwidth of $25 \mathrm{MHz}, 10 \mathrm{MHz}$ and $2 \mathrm{MHz}$, respectively. As clearly demonstrated, a narrower bandwidth filter leads to a lower frequency error and thus a better sensing performance (higher FoM). Since an optimized pump power (at the MI threshold) would achieve a higher SNR and hence a further lower frequency error, a higher FoM value would be expected with an optimized pump power.

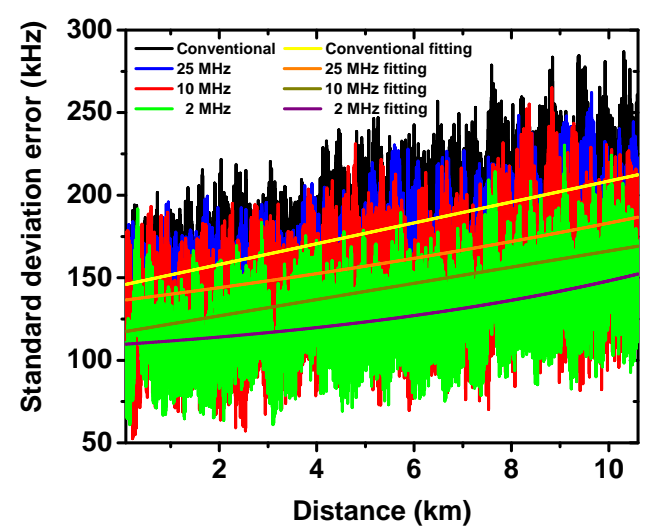

(a)

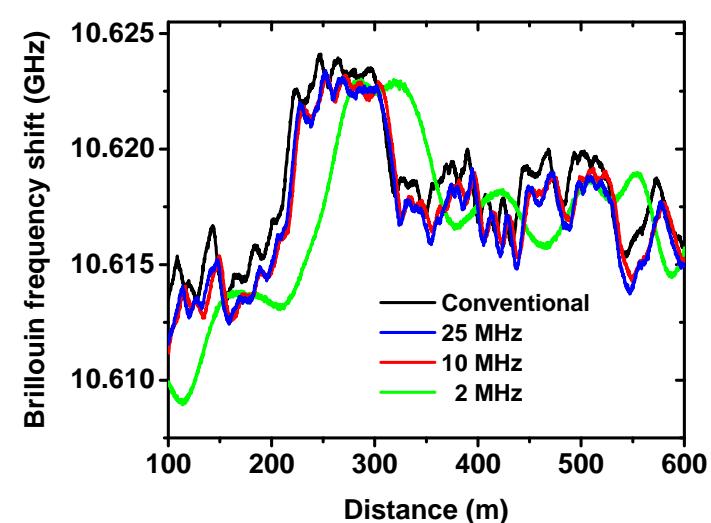

(b)

Figure 5. Experimental measurement using RF lowpass filtering: (a) Standard deviation error versus distance, solid lines represent the exponential fitting; (b) Detection of the strain point.

\section{Discussion}

To further asses the performance of BOTDA with RF filtering, a strain point measurement was conducted. As illustrated in Figure 5b, for all types of filters, the sensor detects the strain point in the near end. However, if the filter bandwidth is too low, as shown by the $2 \mathrm{MHz}$ trace, the detected location of the strain point is shifted, while for the cases with 10 and $25 \mathrm{MHz}$ filter, the trace is almost equally undistorted as that for the conventional case. This shift comes from the group delay at the 
frequency edge of the applied low pass filter. For a high bandwidth filter, only a few frequency components of the trace signal are at the edge of the filter, thus the group delay is negligible and vice versa. According to the data sheet, the $2 \mathrm{MHz}$ filter provides a group delay of about $500 \mathrm{~ns}$ at its edge, which agrees very well with the strain spot shift.

Under the criteria that the step response of the filter should not distort the temporal response of the sensing system, the optimal RF filter bandwidth can also be theoretically estimated. For a BOTDA system with $10 \mathrm{~m}$ spatial resolution achieved by $100 \mathrm{~ns}$ pump pulse, the best noise performance with the lowest trace distortion is optimized with a filter rising time of $1 / 3 \times 100 \approx 33 \mathrm{~ns}$ [34]. Since the relationship between the bandwidth $B$ and the rising time $t_{r}$ for a typical low pass filter is approximately $t_{r} \cdot B \approx 0.35$ [35], the optimal filter bandwidth can be calculated as $10 \mathrm{MHz}$, which shows very good agreement to our simulation as well as experimental results. A filter with a lower bandwidth will further increase the noise performance (clearer traces), but at the cost of trace distortion and measurement error. Therefore, the cut-off frequency has to be selected carefully to balance between noise performance and trace distortion.

Since the bandwidth of the digitizer is narrower than the detector in the experiment, the system bandwidth $B_{s y s}$ is limited to $300 \mathrm{MHz}$ by the analog-to-digital conversion (ADC) process. For a pure AWGN channel, the remained noise level normalized to the conventional case could theoretically reach $8.16 \%, 18.26 \%$ and $28.87 \%$ for a $2 \mathrm{MHz}, 10 \mathrm{MHz}$ and $25 \mathrm{MHz}$ filter, respectively. However, there are numerous practical factors that prevent the noise suppression being that high. The most important factor is that, in a Brillouin amplifier the main noise contribution comes from the Brillouin interaction itself [22,23]. Instead of evenly distributed in system bandwidth, the SBS noise power is gain dependent [22] and thus cannot be significantly reduced with a simple filter. It is believed that the influence of the SBS noise in a distributed Brillouin sensing system, especially with a long pulse width as in the experiment, can hardly be neglected and is of great significance for further investigations. Other factors that limit the noise suppression are not enough samples in the BGS to make the RMS calculation statistically accurate, the steepness of the filter profile, etc.

\section{Conclusions}

In this article, we have presented RF filtering on the detected signal to mitigate the noise level and SNR enhancement in BOTDA sensors. We have theoretically and experimentally demonstrated that the noise level can be reduced by low pass filters, and consequently, the measured standard deviation error of the BFS is reduced. The noise reduction level depends on the employed cut-off frequency of the low pass filter. However, especially for low bandwidths, the noise suppression is not as high as theoretically possible for a pure AWGN channel. This clearly shows the influence of the gain dependent SBS noise on BOTDA sensors, which requires further in detail investigation. The results also indicate that RF filters with too low cut-off frequency lead to a distortion of the trace signals and therefore detection errors for a non-uniform BFS. Instead of an RF filter, a cost effective detector with optimal bandwidth can be used.

Author Contributions: Conceptualization, C.F.; software, S.P.; simulation, C.F.; experimental validation, C.F., J.E.K.; data analysis, C.F., J.E.K.; writing_original draft preparation, J.E.K.; writing—review and editing, C.F., T.S., J.E.K.; visualization, C.F.; supervision, T.S.; funding acquisition, T.S.

Funding: Cheng Feng wishes to acknowledge the financial support from German Research Foundation (DFG SCHN 716/13-1) and Niedersächsisches Vorab (NL—4 Project "QUANOMET"). Jaffar Emad Kadum would like to acknowledge the financial support by Niedersächsisches Vorab (NL-4 Project "QUANOMET") as well as partial funding of Iraqi Ministry of Oil/State Company for Oil Projects (SCOP).

Conflicts of Interest: The authors declare no conflict of interest.

\section{References}

1. Galindez-Jamioy, C.A.; López-Higuera, J.M. Brillouin Distributed Fiber Sensors: An Overview and Applications. J. Sens. 2012, 2012, 1-17. [CrossRef] 
2. Motil, A.; Bergman, A.; Tur, M. [INVITED] State of the art of Brillouin fiber-optic distributed sensing. Opt. Laser Technol. 2016, 78, 81-103. [CrossRef]

3. Bao, Y.; Huang, Y.; Hoehler, M.; Chen, G. Review of Fiber Optic Sensors for Structural Fire Engineering. Sensors 2019, 19, 877. [CrossRef] [PubMed]

4. Meng, D.; Ansari, F.; Feng, X. Detection and monitoring of surface micro-cracks by PPP-BOTDA. Appl. Opt. 2015, 54, 4972-4978. [CrossRef] [PubMed]

5. Kwon, I.B.; Baik, S.J.; Im, K.; Yu, J.W. Development of fiber optic BOTDA sensor for intrusion detection. Sens. Actuators A Phys. 2002, 101, 77-84. [CrossRef]

6. Agrawal, G.P. Nonlinear Fiber Optics, 5th ed.; Elsvier: Amsterdam, The Netherlands, 2013.

7. Horiguchi, T.; Shimizu, K.; Kurashima, T.; Tateda, M.; Koyamada, Y. Development of a distributed sensing technique using Brillouin scattering. J. Light. Technol. 1995, 13, 1296-1302. [CrossRef]

8. Feng, C.; Emad Kadum, J.; Schneider, T. The State-of-the-Art of Brillouin Distributed Fiber Sensing. In Brillouin Distributed and Fiber-Bragg-Grating-Based Fiber Sensing-Principle, Measurement and Applications; IntechOpen: London, UK, 2019.

9. Haneef, S.M.; Yang, Z.; Thévenaz, L.; Venkitesh, D.; Srinivasan, B. Performance analysis of frequency shift estimation techniques in Brillouin distributed fiber sensors. Opt. Express 2018, 26, 14661-14677. [CrossRef]

10. Horiguchi, T.; Tateda, M. Optical-fiber-attenuation investigation using stimulated Brillouin scattering between a pulse and a continuous wave. Opt. Lett. 1989, 14, 408-410. [CrossRef]

11. Soto, M.a.; Thévenaz, L. Modeling and evaluating the performance of Brillouin distributed optical fiber sensors. Opt. Express 2013, 21, 31347-31366. [CrossRef]

12. Alem, M.; Soto, M.A.; Thévenaz, L. Analytical model and experimental verification of the critical power for modulation instability in optical fibers. Opt. Express 2015, 23, 29514-29532. [CrossRef]

13. Thévenaz, L.; Mafang, S.F.; Lin, J. Effect of pulse depletion in a Brillouin optical time-domain analysis system. Opt. Express 2013, 21, 14017-14035. [CrossRef]

14. Lin, W.; Yang, Z.; Hong, X.; Wang, S.; Wu, J. Brillouin gain bandwidth reduction in Brillouin optical time domain analyzers. Opt. Express 2017, 25, 7604-7615. [CrossRef]

15. Rodríguez-Barrios, F.; Martín-López, S.; Carrasco-Sanz, A.; Corredera, P.; Ania-Castañón, J.D.; Thévenaz, L.; González-Herráez, M. Distributed brillouin fiber sensor assisted by first-order raman amplification. J. Light. Technol. 2010, 28, 2162-2172. [CrossRef]

16. Zornoza, A.; Sagues, M.; Loayssa, A. Self-heterodyne detection for SNR improvement and distributed phase-shift measurements in BOTDA. J. Light. Technol. 2012, 30, 1066-1072. [CrossRef]

17. Soto, M.A.; Le Floch, S.; Thévenaz, L. Bipolar optical pulse coding for performance enhancement in BOTDA sensors. Opt. Express 2013, 21, 16390-16397. [CrossRef]

18. Farahani, M.A.; Wylie, M.T.; Castillo-Guerra, E.; Colpitts, B.G. Reduction in the number of averages required in BOTDA sensors using wavelet denoising techniques. J. Light. Technol. 2012, 30, 1134-1142. [CrossRef]

19. Soto, M.A.; Ramírez, J.A.; Thévenaz, L. Intensifying the response of distributed optical fibre sensors using 2D and 3D image restoration. Nat. Commun. 2016, 7, 10870. [CrossRef]

20. Urricelqui, J.; Soto, M.A.; Thévenaz, L. Sources of noise in Brillouin optical time-domain analyzers. In Proceedings of the 24th International Conference on Optical Fibre Sensors, Curitiba, Brazil, 28 September-2 October 2015; Volume 9634, p. 963434.

21. Keiser, G. Optical Fiber Communications, 4th ed.; McGraw-Hill Education (Asia): New York, NY, USA, 2011.

22. Choi, M.; Mayorga, I.C.; Preussler, S.; Schneider, T. Investigation of gain dependent relative intensity noise in fiber brillouin amplification. J. Light. Technol. 2016, 34, 3930-3936. [CrossRef]

23. Wei, W.; Yi, L.; Jaouën, Y.; Morvan, M.; Hu, W. Brillouin Rectangular Optical Filter with Improved Selectivity and Noise Performance. IEEE Photonics Technol. Lett. 2015, 27, 1593-1596. [CrossRef]

24. Zadok, A.; Eyal, A.; Tur, M. Stimulated Brillouin scattering slow light in optical fibers [Invited]. Appl. Opt. 2011, 50, E38. [CrossRef]

25. Preussler, S.; Schneider, T. Stimulated Brillouin scattering gain bandwidth reduction and applications in microwave photonics and optical signal processing. Opt. Eng. 2015, 55, 031110. [CrossRef]

26. Wiatrek, A.; Preußler, S.; Jamshidi, K.; Schneider, T. Frequency domain aperture for the gain bandwidth reduction of stimulated Brillouin scattering. Opt. Lett. 2012, 37, 930-932. [CrossRef]

27. Preussler, S.; Schneider, T. Bandwidth reduction in a multistage Brillouin system. Opt. Lett. 2012, 37, 4122-4124. [CrossRef] 
28. Preussler, S.; Wiatrek, A.; Jamshidi, K.; Schneider, T. Brillouin scattering gain bandwidth reduction down to 3.4 MHz. Opt. Express 2011, 19, 8565-8570. [CrossRef]

29. Zornoza, A.; Olier, D.; Sagues, M.; Loayssa, A. Brillouin distributed sensor using RF shaping of pump pulses. Meas. Sci. Technol. 2010, 21, 094021. [CrossRef]

30. Iribas, H.; Mariñelarena, J.; Feng, C.; Urricelqui, J.; Schneider, T.; Loayssa, A. Effects of pump pulse extinction ratio in Brillouin optical time-domain analysis sensors. Opt. Express 2017, 25, 27896-27911. [CrossRef]

31. Feng, C.; Iribas, H.; Marinelaerña, J.; Schneider, T.; Loayssa, A. Detrimental Effects in Brillouin Distributed Sensors Caused by EDFA Transient. In Proceedings of the 2017 Conference on Lasers and Electro-Optics, San Jose, CA, USA, 14-19 May 2017; p. JTu5A.85.

32. Lecoeuche, V.; Webb, D.J.; Pannell, C.N.; Jackson, D.A. Transient response in high-resolution Brillouin-based distributed sensing using probe pulses shorter than the acoustic relaxation time. Opt. Lett. 2000, 25, 156-158. [CrossRef]

33. Minardo, A.; Bernini, R.; Zeni, L. A simple technique for reducing pump depletion in long-range distributed brillouin fiber sensors. IEEE Sens. J. 2009, 9, 633-634. [CrossRef]

34. Seller, P. Technology Needs for Modular Pixel Detectors. In Solid-State Radiation Detectors: Technology and Applications; Awadalla, S., Ed.; Taylor \& Francis Group: Abingdon, UK, 2015; Chapter 6, pp. 125-142.

35. Orwiler, B. Oscilloscope Vertical Amplifiers, 1st ed.; Tektronix Circuit Concepts: Beaverton, OR, USA,1969; p. 30 .

(c) 2019 by the authors. Licensee MDPI, Basel, Switzerland. This article is an open access article distributed under the terms and conditions of the Creative Commons Attribution (CC BY) license (http://creativecommons.org/licenses/by/4.0/). 